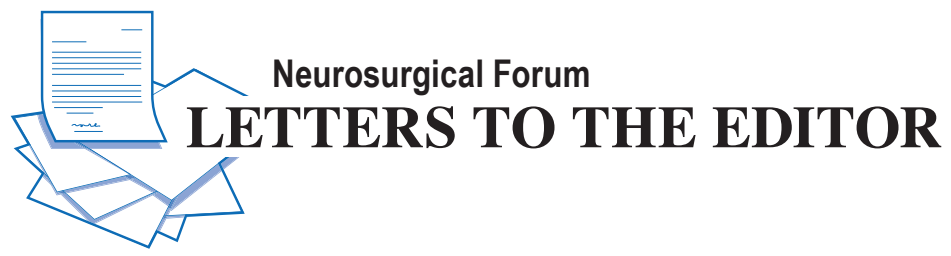

\section{Long-term efficacy of ETV and shunt surgery for management of hydrocephalus}

TO THE EDITOR: We read with interest the article by Beuriat et al. ${ }^{1}$ regarding management of hydrocephalus and its long-term outcome (Beuriat PA, Puget S, Cinalli $\mathrm{G}$, et al: Hydrocephalus treatment in children: long-term outcome in 975 consecutive patients. $J$ Neurosurg Pediatr 20:10-18, July 2017).

We commend the authors for undertaking this study, with one of the longest follow-up periods in a pediatric population with hydrocephalus. The etiologies of hydrocephalus that may impact the long-term efficacy of the treatment and complications of shunt placement have been systematically classified and recorded. However, we would like to bring forth a few issues concerning the article that need further consideration. We observe that this study was undertaken with the aim to understand the preferable treatment modality for hydrocephalus. The two primary modalities studied here were endoscopic third ventriculostomy (ETV) and shunt surgery. In both these treatment groups, the authors noted that there is significant contribution of the etiology of hydrocephalus to determining the long-term efficacy of surgical treatment. We observed in the article's survival curves that different etiologies responded better to the two treatments. Thus, it would be clinically relevant to know which treatment works better for a particular etiology. And consequently, the comparison of the two treatment modalities for each of the common etiologies must have been done to contribute to the clinical decision making. We also note that the ETV and shunt groups differ systematically in terms of the etiology of hydrocephalus. Thus, for an overall comparison of ETV and shunt surgery, a multivariate analysis must have been performed, eliminating the impact of etiology difference.

The classification of etiologies is an elaborate and comprehensive task. However, we find that there are some instances that might be clinically erroneous in spite of being pathologically correct. As an example, cases of postinfectious and posttraumatic hydrocephalus have been clubbed together in the inflammatory group, although the two groups may have a systematic difference in the long-term outcomes in lieu of the etiology. Thus, we suggest that they should have been grouped and analyzed separately and compared with each other. Also, the tumoral group has not been further classified into posterior fossa and supratentorial tumors, and all patients who underwent ETV had posterior fossa tumors, which might also have an impact on efficacy. These errors in classification have resulted in missing out on a few clinically important findings that this study would have contributed.

It is also notable that in the Orbis Sigma Valve (OSV) shunt group the best outcome occurred in those belonging to the "others" group of etiology. It would have been very informative if the authors had mentioned the specific etiologies comprising this group.

The authors have taken note of the fact that there was a systematic difference with regard to the surgeons performing OSV and differential-pressure valve shunt surgeries and also the small number of patients belonging to the differential-pressure valve group, which might be contributing to the differences observed in the long-term outcomes between the two shunts.

In their review of the literature the authors noted a much lower success rate for shunt surgery in other studies compared to their study. ${ }^{2,3}$ However there was no attempt by the authors to explain the probable reasons for these observed differences in the outcome measures.

One of the merits of this study is that it had one of the longest follow-up durations. This merit has not been used to the full extent. The authors noted that several patients required repeat procedures after failure of the first surgery. In understanding the long-term outcomes of the patients with hydrocephalus, it is important to understand not only the rate of failure and need for second surgery but also the outcome of the second surgery. And thus we believe that it would have been beneficial if an analysis of the outcomes of redo ETVs and secondary shunt surgeries after initial ETV or shunt surgery had also been reported.

Additionally, comparison between the efficacy of primary and secondary surgery and comparison between ETV and shunt surgery when performed as the secondary surgery should have been done. 
In conclusion, we would like to say that although this study has undoubtedly highlighted many clinically important findings, it fails to shed light on several important aspects of the topic.

Ravi Sharma, MBBS

Manoj Phalak, MCh

Vivek Tandon, $\mathrm{MCh}$

Ashok K. Mahapatra, MCh

All India Institute of Medical Sciences, New Delhi, India

\section{References}

1. Beuriat PA, Puget S, Cinalli G, Blauwblomme T, Beccaria $\mathrm{K}$, Zerah M, et al: Hydrocephalus treatment in children: long-term outcome in 975 consecutive patients. J Neurosurg Pediatr 20:10-18, 2017

2. Paulsen AH, Lundar T, Lindegaard KF: Twenty-year outcome in young adults with childhood hydrocephalus: assessment of surgical outcome, work participation, and healthrelated quality of life. J Neurosurg Pediatr 6:527-535, 2010

3. Vinchon M, Rekate H, Kulkarni AV: Pediatric hydrocephalus outcomes: a review. Fluids Barriers CNS 9:18, 2012

\section{Disclosures}

The authors report no conflict of interest.

\section{Response}

No response was received from the authors of the original article.

\section{INCLUDE WHEN CITING}

Published online October 27, 2017; DOI: 10.3171/2017.5.PEDS17242.

CAANS 2018, except where prohibited by US copyright law

\section{Biomechanical vulnerability with second concussion}

TO THE EDITOR: I read with interest the article by Post et al. ${ }^{1}$ demonstrating no increase in biomechanical vulnerability among patients who have experienced a repeat concussion compared to those with their first concussion (Post A, Hoshizaki TB, Gilchrist MD, et al: A comparison in a youth population between those with and without a history of concussion using biomechanical reconstruction. J Neurosurg Pediatr 19:502-510, April 2017). They do not give any data on the time between the first and second concussions among the study patients. An article published by my group demonstrated that the time between concussions could be critical. ${ }^{2}$ We found that patients who had a second concussion within 1 year recovered more quickly than patients with a first concussion or those who had a second concussion more than 1 year after their first. We hypothesized that patients who experience head trauma within 1 year of a concussion did have increased vulnerability to a second concussion. Furthermore, patients concussed with less biomechanical force recovered more quickly.

Post et al. could examine the validity of our hypothesis by comparing 3 groups within those patients with repeat concussions: those occurring within 6 months, 6 months to 1 year, and more than a year. I urge them to do so. If on further analysis of their data, they find that patients who suffer a concussion are more vulnerable to a second one within the 1 st year, it would be an important factor in deciding when to allow patients to return to contact sports or other activities in which there is an increased risk of head trauma.

One caveat to consider is that all the patients in their study were recruited from emergency visits. None of our patients with a recurrent concussion within a year presented to an emergency department (ED). They were first seen in our primary care pediatric office. In many of these patients, the chief complaint, unlike most concussed patients, was not head trauma but headache or dizziness. Therefore, if our hypothesis is validated, the physician caring for a patient who has had a concussive episode within a year will need to look for evidence of head trauma and a second concussion even if the chief complaint is not head trauma.

Bruce Taubman, MD

Advocare Cherry Hill Pediatric Group, Cherry Hill, NJ Perelman School of Medicine at the University of Pennsylvania,

Philadelphia, PA

\section{References}

1. Post A, Hoshizaki TB, Gilchrist MD, Koncan D, Dawson L, Chen W, et al: A comparison in a youth population between those with and without a history of concussion using biomechanical reconstruction. J Neurosurg Pediatr 19:502-510, 2017

2. Taubman B, McHugh J, Rosen F, Elci OU: Repeat concussion and recovery time in a primary care pediatric office. J Child Neurol 31:1607-1610, 2016

\section{Disclosures}

The author reports no conflict of interest.

\section{Response}

We thank Dr. Taubman for his interest in this research paper. We read with interest the paper that his group published examining the recovery time for patients with a history, or no history, of concussion. The differences in the results between the Dr. Taubman's paper and this work may be reflected in differences in methods, specifically the subject sample and the dependent variables. The data set from our paper included a pediatric cohort that presented to tertiary pediatric EDs, whereas in the article by Taubman et al., ${ }^{5}$ the study included participants who presented to primary care clinics. This difference in the populations might affect a comparison of results given the possibility that the ED population may have presented with greater initial symptom burden or more severe mechanism of injury. Additionally, Taubman et al. ${ }^{5}$ focused on the recovery of the patients, using neurocognitive testing, and how previous concussions can affect this outcome. In our study we focused on dependent variables that were entirely biomechanical in nature, with no quantification of recovery time based on previous head injury history. We concur that it would be valuable in the future to examine 
the relationship between the biomechanics of impact and the association with neurocognitive outcomes and recovery times in pediatric concussion.

We also wanted to address the comment regarding the timing between the first and second concussions. Since many participants included in our analysis had more than 2 concussions, we defined the historical group as 1 or more concussions. Given that the objective of our study was to ascertain if the vulnerability of brain tissues would be evident using biomechanical reconstruction analysis, the anatomical and tissue research would support Dr. Taubman's hypothesis that there would be a vulnerability within a given time frame following a concussion and also that lower biomechanical forces might affect recovery time. ${ }^{1-4}$ As a result, windows of vulnerability related to tissue responses can be measured post-impact. Unfortunately, in reconstruction work, the magnitude of impact that created the concussion was not controlled. As a result it may be that the youth impacted in this study would have received a concussion regardless of the tissue's vulnerability, since the event was of such severity that a brain injury would have occurred regardless. This may have increased the likelihood that there would be no observed difference between the history and no-history groups as described in the paper.

\begin{abstract}
Andrew Post, $\mathrm{PhD}^{1,2}$ T. Blaine Hoshizaki, PhD² Michael D. Gilchrist, PhD $^{3}$ David Koncan, MSc ${ }^{2}$ Lauren Dawson, MSc ${ }^{2}$ Wesley Chen, BSc ${ }^{2}$ Andrée-Anne Ledoux, $\mathrm{PhD}^{1}$ Roger Zemek, MD1 Pediatric Emergency Research Canada (PERC) 5P Concussion Team

${ }^{1}$ Children's Hospital of Eastern Ontario Research Institute, Ottawa, ON, Canada

${ }^{2}$ Human Kinetics, University of Ottawa, Ottawa, ON, Canada ${ }^{3}$ School of Mechanical \& Materials Engineering, University College,
\end{abstract} Dublin, Ireland

\section{Appendix}

The collaborators for the PERC 5P Concussion Team were as follows: Candice McGahern, BA; Gurinder Sangha, MD; Darcy Beer, MD; William Craig, MDCM; Ken J. Farion, MD; Angelo Mikrogianakis, MD; Karen Barlow, MD; Alexander S. Dubrovsky, MDCM, MSc; Willem Meeuwisse, MD, PhD; William P. Meehan III, MD; Yael Kamil, BSc; Anne M. Grool, MD, PhD, MSc; Blaine Hoshizaki, PhD; Peter Anderson, PhD; Brian L. Brooks, PhD; Michael Vassilyadi, MDCM, MSc; Terry Klassen, MD; Michelle Keightley, PhD; Lawrence Richer, MD; and Carol DeMatteo, MSc.

\section{References}

1. Effgen GB, Morrison B III: Electrophysiological and pathological characterization of the period of heightened vulnerability to repetitive injury in an in vitro stretch model. J Neurotrauma 34:914-924, 2017

2. Friess SH, Ichord RN, Ralston J, Ryall K, Helfaer MA, Smith C, et al: Repeated traumatic brain injury affects composite cognitive function in piglets. J Neurotrauma 26(7):11111121,2009
3. Prins ML, Alexander D, Giza CC, Hovda DA: Repeated mild traumatic brain injury: Mechanisms of cerebral vulnerability. J Neurotrauma 30:30-38, 2013

4. Slemmer JE, Weber JT: The extent of damage following repeated injury to cultured hippocampal cells is dependent on the severity of insult and inter-injury interval. Neurobiol Dis 18:421-431, 2005

5. Taubman B, McHugh J, Rosen F, Elci OU: Repeat concussion and recovery time in a primary care pediatric office. J Child Neuro 31:1607-1610, 2016

\section{INCLUDE WHEN CITING}

Published online October 27, 2017; DOI: 10.3171/2017.5.PEDS17231.

CAANS 2018, except where prohibited by US copyright law

\section{Posterior fossa tumor resection and the failure of endoscopic third ventriculostomy and ventriculoperitoneal shunts}

TO THE EDITOR: I read with interest the recent article by Dewan et al. ${ }^{3}$ (Dewan MC, Lim J, Shannon CN, et al: The durability of endoscopic third ventriculostomy and ventriculoperitoneal shunts in children with hydrocephalus following posterior fossa tumor resection: a systematic review and time-to-failure analysis. J Neurosurg Pediatr 19:578-584, May 2017). I wish to commend the authors for this work involving both a systematic selection and review of 12 publications indexed in PubMed and a retrospective review of their own series of posterior fossa brain tumor (PFBT) patients requiring CSF diversion. They report that endoscopic third ventriculostomy (ETV) failure occurred sooner than ventriculoperitoneal shunt (VPS) failure, that long-term treatment durability was found to be higher for ETV, and that complications occurred more frequently with VPS than with ETV. Although they mention that postoperatively about $30 \%$ of patients will have persistent hydrocephalus requiring permanent CSF diversion, the authors do not clearly state whether all 12 studies included in the analysis strictly examined only postoperative cases of hydrocephalus occurring after excision of PFBT, or whether some of the studies also included patients treated for hydrocephalus prior to PFBT excision or persisting after surgery. Instead, they simply mention that the analysis between ETV and VPS involved patients treated for hydrocephalus related to PFBT, both for the studies from the systematic review and for their own retrospective series. ${ }^{3}$

There are a few logical reasons to support recommending ETV for patients with hydrocephalus arising from lowgrade lesions, who have a probability of longer survival, and VPS for hydrocephalus occurring as a result of the more aggressive PFBT, with limited survival. ${ }^{3}$ Endoscopic third ventriculostomy has been suggested as the procedure of choice following postoperative cases of hydrocephalus over VPS, though there is still insufficient information from the literature to support this., ${ }^{2,4,6,7}$ Some studies have confirmed the high success rate and low complication rate of ETV. ${ }^{5}$ One series reported that ETV successfully controlled all 8 cases of post-PFBT excision hydrocepha- 
lus, even when there was already subarachnoid seeding at the time of ETV.? Progressive hydrocephalus as a result of subsequent shunt failure in 3 patients in another series was successfully managed with ETV. ${ }^{2}$ Furthermore, when done prior to PFBT resection, ETV has been shown to significantly reduce the risk of postoperative hydrocephalus. ${ }^{1,7}$ These benefits are important for patients with PFBT in whom longer survival is anticipated.

On the other hand, the risk, commonly associated with VPS, of peritoneal seeding via the CSF may not really be a significant problem as a possible complication of CSF diversion for hydrocephalus following resection of more aggressive lesions because of the shorter life expectancy often associated with such lesions. Moreover, even though VPS has a greater tendency for complications than ETV, as clearly demonstrated, the shorter life expectancy characteristic of the recurrence of more aggressive lesions is often not likely to provide sufficient time for any of the common VPS complications to occur before the death of the patient from tumor progression. However, as the authors rightly mention, issues like these remain quite uncertain and definitely require multi-institutional collaboration and thoughtful study design to be objectively confirmed.

\section{Chiazor U. Onyia, MBBS}

Obafemi Awolowo University Teaching Hospitals Complex, lle-lfe, Nigeria

\section{References}

1. Al Qroom R, Khasawneh N, Haddad F, Alqurashi M: Neuroendoscopy sway in the treatment of posterior fossa tumours associated hydrocephalus in children. J Neurol Stroke 6:00203, 2017

2. Cinalli G, Salazar C, Mallucci C, Yada JZ, Zerah M, SainteRose C: The role of endoscopic third ventriculostomy in the management of shunt malfunction. Neurosurgery 43:13231329,1998

3. Dewan MC, Lim J, Shannon CN, Wellons JC III: The durability of endoscopic third ventriculostomy and ventriculoperitoneal shunts in children with hydrocephalus following posterior fossa tumor resection: a systematic review and time-to-failure analysis. J Neurosurg Pediatr 19:578-584, 2017

4. Jones RF, Stening WA, Brydon M: Endoscopic third ventriculostomy. Neurosurgery 26:86-92, 1990

5. Oertel JMK, Baldauf J, Schroeder HWS, Gaab MR: Endoscopic options in children: experience with 134 procedures. J Neurosurg Pediatr 3:81-89, 2009

6. Rehman Z, Khanzada K, Husain R, Ali M, Ali M: Experience with endoscopic third ventriculostomy in the management of hydrocephalus secondary to posterior fossa tumor. $\mathbf{J}$ Postgrad Med Inst 27:433-438, 2013

7. Sainte-Rose C, Cinalli G, Roux FE, Maixner R, Chumas PD, Mansour M, et al: Management of hydrocephalus in pediatric patients with posterior fossa tumors: the role of endoscopic third ventriculostomy. J Neurosurg 95:791-797, 2001

\section{Disclosures}

The author reports no conflict of interest.

\section{Response}

We appreciate the thoughtful response offered by Dr. Onyia. This is an important topic, and fruitful discourse is needed if we are to move closer to definitive answers.

Regarding the query posed in the letter, our systematic review did not stipulate a specific timing of CSF diversion relative to PFBT resection. As mentioned among our study's limitations, many source articles did not specify relative timing for individuals. Particularly in mixedcohort studies (those describing both pre- and postresection ETV), survival data often could not be disaggregated. Thus, in an effort to maximize ETV patients and strengthen the comparison, preresection ETV was not an exclusion criterion. Is it then possible that some patients in the metaregressed ETV cohort underwent CSF diversion unnecessarily, as they would not have been destined to persistent hydrocephalus? This is indeed possible. It is why we were careful to remind the reader that our results should be interpreted as a summary of published data, rather than as definitive evidence to pursue one treatment modality over the other. Bias and dissimilar comparator groups are just two reasons why practice standards are best not built on retrospective uncontrolled analyses.

It is relevant to note that at our institution, preresection ETV is never performed, as we believe that doing so would unnecessarily expose the majority of patients who do not develop persistent hydrocephalus to the risks of an invasive procedure. This is all despite the low risk profile of ETV, which is illustrated in our systematic review.

Dr. Onyia has echoed several of the most important concepts underlying the decision to treat PFBT-related hydrocephalus with ETV or VPS. Among them he refers to the differential survival duration based on tumor grade, the risk of subarachnoid and peritoneal seeding, and, of course, the success rates reported by other surgeons. So while there are many factors available to guide today's neurosurgeon in this treatment decision, one of the most important is absent: high-quality evidence. Fortunately, unlike many clinical variables that are not modifiable, as a community of neurosurgeons we have the ability to change this. Our study suggests that equipoise exists, and a randomized clinical trial comparing ETV and VPS in these patients seems not only sensible, but necessary.

Michael C. Dewan, MD, MSCI John C. Wellons III, MD, MSPH

Vanderbilt University Medical School, Nashville, TN

\section{INCLUDE WHEN CITING}

Published online November 3, 2017; DOI: 10.3171/2017.6.PEDS17283.

CAANS 2018, except where prohibited by US copyright law 